\title{
ON NUMERICAL SOLUTION OF MULTIPARAMETER STURM-LIOUVILLE SPECTRAL PROBLEMS
}

\author{
T. LEVITINA \\ Computing Center, Russian Academy of Sciences \\ Vavilova 40, 117967 Moscow GSP-1, Russia \\ E-mail: LEV@SMS.CCAS.MSK.SU
}

The method proposed here has been devised for solution of the spectral problem for the Lamé wave equation (see [2]), but extended lately to more general problems. This method is based on the phase function concept or the Prüfer angle determined by the Prüfer transformation $\cot \theta(x)=y^{\prime}(x) / y(x)$, where $y(x)$ is a solution of a second order self-adjoint o.d.e. The Prüfer angle $\theta(x)$ has some useful properties very often being referred to in theoretical research concerning both single- and multi-parameter Sturm-Liouville spectral problems (see e.g. $[6,14,5]$ ). All these properties may be useful for numerical solution of the above problems as well. For an account of numerical methods for solving the single-parameter Sturm-Liouville spectral problem by means of a modified Prüfer transformation one is referred to $[1,11,9]$.

1. First, we shall consider the case of the following couple of second order self-adjoint o.d.e. with coefficients depending linearly on two spectral parameters:

$$
\begin{cases}y_{1}^{\prime \prime}+\left(q_{1}\left(x_{1}\right)+\lambda p_{11}\left(x_{1}\right)+\mu p_{12}\left(x_{1}\right)\right) y_{1}=0, & x_{1} \in[0,1] \\ y_{2}^{\prime \prime}+\left(q_{2}\left(x_{2}\right)+\lambda p_{21}\left(x_{2}\right)+\mu p_{22}\left(x_{2}\right)\right) y_{2}=0, & x_{2} \in[0,1]\end{cases}
$$

each of the functions $y_{1}, y_{2}$ is subject to the homogeneous boundary conditions

$$
\left\{\begin{array}{l}
\nu_{i 0}^{-1} y_{i}^{\prime}(0) \sin \alpha_{i}+\nu_{i 0} y_{i}(0) \cos \alpha_{i}=0, \\
\nu_{i 1}^{-1} y_{i}^{\prime}(1) \sin \beta_{i}+\nu_{i 1} y_{i}(1) \cos \beta_{i}=0,
\end{array} \quad i=1,2 .\right.
$$

Here we have introduced positive numbers $\nu_{i j}$ to be clarified later on.

1991 Mathematics Subject Classification: Primary 65L15.

The paper is in final form and no version of it will be published elsewhere. 
In order to use the known theoretical results without any additional comments we shall assume everywhere that all the coefficients in the given equations are continuous and even sufficiently smooth. (Note that most of the results are true under essentially weaker restrictions - see e.g. [5]. The consideration of this question is outside the scope of the article.)

Suppose that for each $x_{1}, x_{2} \in I$,

$$
p_{11}\left(x_{1}\right)>0, \quad p_{12}\left(x_{1}\right)>0, \quad p_{21}\left(x_{2}\right)>0, \quad p_{22}\left(x_{2}\right)<0
$$

(here $I=[0,1])$. According to standard well-known results in Sturm-Liouville's theory for a single equation the conditions (3) yield the following monotonicity of the so-called spectral curves $\mu=\mu_{i}^{n_{i}}(\lambda), i=1,2: \mu_{1}^{n_{1}}(\lambda)$ decreases in $\lambda$ while $\mu_{2}^{n_{2}}(\lambda)$ increases. Here we denote by $\mu=\mu_{i}^{n_{i}}(\lambda)$ the eigenvalue $\mu$ associated with the reduced Sturm-Liouville problem restricted to the $i$ th equation and $i$ th pair of boundary conditions. The index $n_{i}$ means that the corresponding eigenfunction $y_{i}^{n_{i}}\left(x_{i}\right)$ has exactly $n_{i}$ internal zeros. Both mappings $\mu=\mu_{i}^{n_{i}}(\lambda)$, $i=1,2$, are analytic (see [5]) and one-to-one, so they have to intersect at a single point $\left(\lambda^{n}, \mu^{n}\right)$ which is the eigenvalue of the complete problem (1)-(2) with eigenfunction $Y^{n}\left(x_{1}, x_{2}\right)=y_{1}^{n_{1}}\left(x_{1}\right) \times y_{2}^{n_{2}}\left(x_{2}\right), n=\left(n_{1}, n_{2}\right)$.

2. The spectral curves divide the plane $(\lambda, \mu)$ of spectral parameters into four regions, each characterized by the signs of the differences $\Delta \mu_{i}=\mu-\mu_{i}^{n_{i}}(\lambda)$ and $\Delta \lambda_{i}=\lambda-\lambda_{i}^{n_{i}}(\mu)$ :

region 1: $\Delta \mu_{1}<0$ and $\Delta \mu_{2}>0, \quad \Delta \lambda_{1}<0$ and $\Delta \lambda_{2}<0, \quad$ hence $\lambda<\lambda^{n}$; region 2: $\quad \Delta \mu_{1}<0$ and $\Delta \mu_{2}<0, \quad \Delta \lambda_{1}<0$ and $\Delta \lambda_{2}>0, \quad$ hence $\mu<\mu^{n}$; region 3: $\Delta \mu_{1}>0$ and $\Delta \mu_{2}<0, \quad \Delta \lambda_{1}>0$ and $\Delta \lambda_{2}>0, \quad$ hence $\lambda>\lambda^{n}$;

region 4: $\Delta \mu_{1}>0$ and $\Delta \mu_{2}>0, \Delta \lambda_{1}>0$ and $\Delta \lambda_{2}<0, \quad$ hence $\mu>\mu^{n}$.

To find the position of an arbitrary point $(\lambda, \mu)$ with respect to the eigenvalue $\left(\lambda^{n}, \mu^{n}\right)$, the modified phase functions $\theta_{i}$ are introduced:

$$
\frac{y_{i}^{\prime}\left(x_{i}\right)}{y_{i}\left(x_{i}\right)}=\nu_{i}^{2}\left(x_{i}\right) \cot \theta_{i}\left(x_{i}, \lambda, \mu\right), \quad i=1,2 .
$$

Here $\nu_{i}\left(x_{i}\right)$ is the so-called scaling function. We use scaling functions to make the Prüfer angle change as slowly as possible. If neither equation of system (1) has a turning point within $I$, then the scaling functions should be chosen to satisfy $\nu_{i}^{2}\left(x_{i}\right)=\sqrt{q_{i}\left(x_{i}\right)+\lambda p_{i 1}\left(x_{i}\right)+\mu p_{i 2}\left(x_{i}\right)}$; otherwise the choice of $\nu_{i}\left(x_{i}\right)$ is a more sophisticated task. Anyway, for the sake of simplicity one could set $\nu_{i}\left(x_{i}\right) \equiv 1$.

Since the functions $y_{i}\left(x_{i}\right)$ are solutions of $(1)$, the phase functions satisfy the first order non-linear phase equations

$$
\begin{array}{r}
\theta_{i}^{\prime}=\nu_{i}^{2}\left(x_{i}\right) \cos ^{2} \theta_{i}+\frac{q_{i}\left(x_{i}\right)+\lambda p_{i 1}\left(x_{i}\right)+\mu p_{i 2}\left(x_{i}\right)}{\nu_{i}^{2}\left(x_{i}\right)} \sin ^{2} \theta_{i}+\frac{\nu_{i}\left(x_{i}\right)^{\prime}}{\nu_{i}\left(x_{i}\right)} \sin 2 \theta_{i}, \\
x_{i} \in I, \quad i=1,2 .
\end{array}
$$


If the solution $y_{i}\left(x_{i}\right)$ satisfies the $i$ th left boundary condition (2) then the phase function $\theta_{i}^{l}$ is initially equal to $\alpha_{i}$ :

$$
\theta_{i}^{l}(0, \lambda, \mu)=\alpha_{i},
$$

and referred to as the left-defined phase function $\theta_{i}^{l}\left(x_{i}, \lambda, \mu\right)$. In contrast to the left-defined phase function (6), the right-defined one, determined by

$$
\theta_{i}^{r}(1, \lambda, \mu)=\beta_{i}+\pi n_{i},
$$

accounts for the number of oscillations of each solution $y_{i}\left(x_{i}\right)$ associated with it (see $[6,9])$.

For each fixed pair $(\lambda, \mu)$ let us compute both $\theta_{i}^{l}(x, \lambda, \mu)$ and $\theta_{i}^{r}(x, \lambda, \mu)$ as the solutions of the initial value problems (5), (6) and (5), (7), respectively. The signs of the differences

$$
\Delta \theta_{i}=\theta_{i}^{l}\left(x_{i}^{*}, \lambda, \mu\right)-\theta_{i}^{r}\left(x_{i}^{*}, \lambda, \mu\right)
$$

at an arbitrary point $x_{i}^{*}$ coincide with the signs of $\Delta \lambda_{i}, i=1,2$. Moreover, $\Delta \theta_{i}=0$ iff the point $(\lambda, \mu)$ lies on the curve $\mu=\mu_{i}^{n_{i}}(\lambda)$.

Thus the two-parameter spectral problem (1)-(2) is reduced to the system of equations

$$
\left\{\begin{array}{l}
\Delta \theta_{1}\left(x_{1}^{*}, \lambda, \mu\right)=0 \\
\Delta \theta_{2}\left(x_{2}^{*}, \lambda, \mu\right)=0
\end{array}\right.
$$

with functions $\Delta \theta_{i}$ having the above properties.

The algorithm of numerical solution of the problem above consists of two steps. The first one is the construction of a rectangle containing the eigenvalue, i.e. we have to find one point for each region and draw a vertical line if the point $(\lambda, \mu)$ lies in region 1 or 3 and a horizontal one otherwise.

After this rectangle has been constructed, the iterative process begins. At each step, the rectangle is divided evenly either by a vertical or a horizontal line depending on whether the center of the current rectangle lies in regions 1 and 3 or in 2 and 4 respectively. Then the half of the rectangle not containing the eigenvalue is ignored. Note that each time the decision is made only by means of the solutions of the initial value problems (5), (6) and (5), (7).

No matter what the initial guess is, the process tends to the eigenvalue.

Using phase functions and some additional auxiliary functions there exists a method to recover the corresponding eigenfuction normalized (for example) by

$$
\iint_{I^{2}}\left\{p_{11}\left(x_{1}\right) p_{22}\left(x_{2}\right)-p_{12}\left(x_{1}\right) p_{21}\left(x_{2}\right)\right\} Y\left(x_{1}, x_{2}\right) d x_{1} d x_{2}=1 .
$$

3. Before proceeding to the above algorithm we have made strong assumptions (3) on the behavior of coefficients. These restrictions can be relaxed. Instead of (3) it is sufficient to assume that

$$
\operatorname{det}\left\{p_{i j}\left(x_{i}\right)\right\}=p_{11}\left(x_{1}\right) p_{22}\left(x_{2}\right)-p_{12}\left(x_{1}\right) p_{21}\left(x_{2}\right) \neq 0 \quad \text { for all }\left(x_{1}, x_{2}\right) \in I^{2} .
$$


(Note that the sign of the determinant does not change by the continuity of $p_{i j}$ and (8).) Then there is a non-singular linear transformation of the spectral parameters

$$
\lambda=a_{1} \nu+b_{1} \eta, \quad \mu=a_{2} \nu+b_{2} \eta,
$$

such that the new coefficients $\widetilde{p}_{i j}$ defined by

$$
\left(\begin{array}{ll}
\widetilde{p}_{11} & \widetilde{p}_{12} \\
\widetilde{p}_{21} & \widetilde{p}_{22}
\end{array}\right)=\left(\begin{array}{ll}
p_{11} & p_{12} \\
p_{21} & p_{22}
\end{array}\right)\left(\begin{array}{ll}
a_{1} & b_{1} \\
a_{2} & b_{2}
\end{array}\right)
$$

satisfy (3). This fact was proved for an arbitrary number of spectral parameters by Sleeman in [13]. In [10] another proof was proposed for the two-parameter case, which enables us to choose new spectral parameters explicitly. The inequality (8) is just the sufficient condition for the validity of Klein's oscillation theorem (see $[7])$, i.e. the problem (1)-(2) has discrete spectrum and the eigenfunctions form a complete set in $\mathcal{L}_{2}\left[I^{2}\right]$, orthogonal with respect to the weight

$$
\sigma\left(x_{1}, x_{2}\right)=p_{11}\left(x_{1}\right) p_{22}\left(x_{2}\right)-p_{12}\left(x_{1}\right) p_{21}\left(x_{2}\right) .
$$

The described algorithm can be applied to equations with non-linear coefficients in spectral parameters. Instead of (1) we shall consider the system

$$
\begin{cases}y_{1}^{\prime \prime}+Q_{1}\left(x_{1}, \lambda, \mu\right) y_{1}=0, & x_{1} \in I, \\ y_{2}^{\prime \prime}+Q_{2}\left(x_{2}, \lambda, \mu\right) y_{2}=0, & x_{2} \in I,\end{cases}
$$

with boundary conditions (2).

Suppose each reduced problem in (10), (2) can be solved for any fixed $\lambda$ with respect to $\mu$ and vice versa. If $Q_{1}\left(x_{1}, \lambda, \mu\right)$ increases in both $\lambda$ and $\mu$, and $Q_{2}\left(x_{2}, \lambda, \mu\right)$ increases in $\lambda$ and decreases in $\mu$, one can apply the algorithm without any changes because the spectral curves have the same monotonicity as in the linear case. This analogy can be continued as follows. Let $K_{1}$ be the set such that each line $\mu-\mu_{0}=k\left(\lambda-\lambda_{0}\right)$ intersects the first spectral curve at a single point $\left(\lambda_{0}, \mu_{0}\right)$; here $k \in K_{1}$ and $\mu_{0}=\mu_{1}^{n_{1}}\left(\lambda_{0}\right)$. The set $K_{2}$ is defined in the same way. We introduce another two sets: $M_{1}=\overline{\mathbb{R} \backslash K_{1}}, M_{2}=\overline{\mathbb{R} \backslash K_{2}}$. Note that for each point $\left(\lambda_{0}, \mu_{0}\right)$ of the $i$ th spectral curve the pair of sectors formed by the lines $\mu-\mu_{0}=m\left(\lambda-\lambda_{0}\right)$ with $m \in M_{i}$ covers the whole curve $\mu=\mu_{i}^{n_{i}}(\lambda)$. If

$$
M_{1} \cap M_{2}=\emptyset
$$

then the new spectral parameters may be chosen by means of (9) in which the functions $\widetilde{Q}_{i}(x, \nu, \eta)=Q_{i}(x, \lambda, \mu)$ have the required monotonicity. In the linear case the condition (11) is equivalent to (8).

If the sets $M_{1}, M_{2}$ have a common bound $l$ and this bound lies in both $K_{1}$ and $K_{2}$, then the line $\mu=l \lambda$ determines the new coordinate direction.

The bounds of the sets $M_{1}, M_{2}$ may be defined from the known estimates for the derivatives of the spectral curves $d \mu_{i} / d \lambda \in\left[k_{1 i}, k_{2 i}\right]$ like in $[5,12]$, where

$$
k_{1 i}=-\max _{[0,1]}\left[\frac{\partial Q_{i}}{\partial \lambda} / \frac{\partial Q_{i}}{\partial \mu}\right], \quad k_{2 i}=-\min _{[0,1]}\left[\frac{\partial Q_{i}}{\partial \lambda} / \frac{\partial Q_{i}}{\partial \mu}\right] .
$$


4. The method may be extended to the case of three spectral parameters. We shall consider the system

$$
\begin{cases}y_{1}^{\prime \prime}+\left(q_{1}\left(x_{1}\right)+\lambda p_{11}\left(x_{1}\right)+\mu p_{12}\left(x_{1}\right)+\nu p_{13}\left(x_{1}\right)\right) y_{1}=0, & x_{1} \in I, \\ y_{2}^{\prime \prime}+\left(q_{2}\left(x_{2}\right)+\lambda p_{21}\left(x_{2}\right)+\mu p_{22}\left(x_{2}\right)+\nu p_{23}\left(x_{2}\right)\right) y_{2}=0, & x_{2} \in I, \\ y_{3}^{\prime \prime}+\left(q_{3}\left(x_{3}\right)+\lambda p_{31}\left(x_{3}\right)+\mu p_{32}\left(x_{3}\right)+\nu p_{33}\left(x_{3}\right)\right) y_{3}=0, & x_{3} \in I,\end{cases}
$$

with boundary conditions (2) where $i=1,2,3$. It was proved in [13] that if

$$
\operatorname{det}\left\{p_{i j}\left(x_{i}\right)\right\} \neq 0
$$

for any $\left(x_{1}, x_{2}, x_{3}\right) \in I^{3}$ with $i, j=1,2,3$, then there exists a non-singular linear transformation of the spectral parameters which provides the desired signs of new coefficients. So we shall restrict our consideration to the coefficients with signs

$$
\left(\begin{array}{ccc}
+ & + & + \\
+ & + & - \\
+ & - & -
\end{array}\right)
$$

Suppose also that all the second order minors of $\left(p_{i j}\left(x_{i}\right)\right)$ do not vanish.

Further, (12) leads to the existence of the solution $\left(\lambda^{n}, \mu^{n}, \nu^{n}\right)$ of this threeparameter spectral problem for an arbitrary triplet $n=\left(n_{1}, n_{2}, n_{3}\right)$, where $n_{1}, n_{2}$, $n_{3}$ are the numbers of internal zeros of $y_{1}\left(x_{1}\right), y_{2}\left(x_{2}\right), y_{3}\left(x_{3}\right)$ respectively (Klein's oscillation theorem). This means that the spectral surfaces $\nu=\nu_{i}^{n_{i}}(\lambda, \mu), i=$ $1,2,3$, divide the spectral space into eight regions.

Thus, as in the two-parameter case, each point of the spectral space is associated with the three signs of the differences of the phase functions, given by (4)-(8) where $i=1,2,3$. Moving from an arbitrary point $(\lambda, \mu, \nu)$ along the coordinate lines one may determine a point in regions with signs

$$
\begin{array}{ll}
(+,+,+), & \text { where } \lambda>\lambda^{n}, \\
(-,-,-), & \text { where } \lambda<\lambda^{n}, \\
(+,+,-), & \text { where } \mu>\mu^{n}, \\
(-,-,+), & \text { where } \mu<\mu^{n}, \\
(+,-,-), & \text { where } \nu>\nu^{n}, \\
(-,-,+), & \text { where } \nu<\nu^{n} .
\end{array}
$$

The planes $\lambda=$ const for the first two points, $\mu=$ const for the next ones, and $\nu=$ const for the last form a parallelepiped containing the spectral point.

If the center $\left(\lambda^{*}, \mu^{*}, \nu^{*}\right)$ of the current parallelepiped belongs to one of the regions above, then just like in the two-parameter case, the appropriate coordinate plane cuts off half of the parallelepiped with the eigenvalue, while the other half is ignored. For instance, if the point $\left(\lambda^{*}, \mu^{*}, \nu^{*}\right)$ is in the region $(+,+,+)$ then the eigenvalue is located in the half $\lambda<\lambda^{*}$. However, there is a difference between the two- and three-parameter cases. The point is that there are two regions

$$
(+,-,+) \text { and }(-,+,-)
$$


with boundaries intersecting twice the coordinate lines or having no common point with these lines at all. So if the center $\left(\lambda^{*}, \mu^{*}, \nu^{*}\right)$ lies in these regions, the position of the eigenvalue with respect to each coordinate plane is not known a priori.

To overcome this drawback we propose the following. Let $\left(\lambda^{*}, \mu^{*}, \nu^{*}\right)$ be in the region $(+,-,+)$. Moving along the coordinate line $\lambda$ in the direction of its decrease, we eventually find a point $\left(\lambda^{* *}, \mu^{*}, \nu^{*}\right)$ belonging to the region $(-,-,-)$. But within the interval $\left[\left(\lambda^{*}, \mu^{*}, \nu^{*}\right),\left(\lambda^{* *}, \mu^{*}, \nu^{*}\right)\right]$ there have to be points either from the region $(-,-,+)$ or from $(+,-,-)$. After any of these points is found (e.g. by successive bisection of the interval) one should draw the plane $\mu=\mu^{*}$ or $\nu=\nu^{*}$ depending on the region where this point happens to be. The eigenvalue lies in the half $\mu<\mu^{*}$ or $\nu>\nu^{*}$ respectively.

The division of the current parallelepiped should be continued until the current parallelepiped containing the eigenvalue becomes sufficiently small.

It is worth mentioning that the isolation of the eigenvalue in an $n$-dimensional parallelepiped should be done in the same way provided (12) holds for any $\left(x_{1}, \ldots, x_{n}\right) \in I^{n}$. However, the greater the value of $n$, the more complicated the localization of the eigenvalue, since the number of regions in the spectral parameter space grows faster than the number of coordinate semi-axes.

The described algorithm was successfully applied to the two-parameter Lamé wave equation for a wide range of problem data changes. Up till now only a few calculations of the ellipsoidal wave functions are known $([3,4])$. For instance, standard programs based upon this algorithm solve the problem of scattering of plane waves on a general ellipsoid. (The analytical solution in the form of a series of ellipsoidal wave functions was proposed in [8].)

The author is grateful to A. A. Abramov and N. B. Konyukhova for the discussion of the scope of the paper and for useful remarks.

\section{References}

[1] A. A. Abramov, Methods of solution of some linear problems, doctoral dissertation, Computing Center Acad. Sci. USSR, Moscow 1974 (in Russian).

[2] A. A. Abramov, A. L. Dyshko, N. B. Konyukhova and T. V. Levitina, Computation of angular wave functions of Lamé by means of solution of auxiliary differential equations, Zh. Vychisl. Mat. i Mat. Fiz. 29 (6) (1989), 813-830 (in Russian); English transl.: USSR Comput. Math. and Math. Phys. 29 (1989).

[3] F. M. Arscott and B. D. Sleeman, High-frequency approximations to ellipsoidal wave functions, Mathematika 17 (1970), 39-46.

[4] F. M. Arscott, P. J. Taylor and R. V. M. Zahar, On the numerical construction of ellipsoidal wave functions, Math. Comp. 40 (1983), 367-380.

[5] P. A. Binding and P. J. Browne, Asymptotics of eigencurves for second order ordinary differential equations, part I, J. Differential Equations 88 (1990), 30-45, part II, ibid. 89 (1991), 224-243. 
[6] E. A. Coddington and N. Levinson, Theory of Ordinary Differential Equations, McGraw-Hill, New York 1955.

[7] M. Faerman, The completeness and expansion theorem associated with multiparameter eigenvalue problem in ordinary differential equations, J. Differential Equations 5 (1969), 197-213.

[8] M. V. Fedoryuk, Diffraction of waves by a tri-axial ellipsoid, Differentsial'nye Uravneniya 25 (11) (1989), 1990-1995 (in Russian).

[9] D. I. Kitoroagè, N. V. Konyukhova and B. S. Pariǔskiŭ, A Modified Phase Function Method for Problems Concerning Bound States of Particles, Soobshch. Prikl. Mat., Vychisl. Tsentr Akad. Nauk SSSR, Moscow 1986 (in Russian).

[10] T. V. Levitina, Conditions of applicability of an algorithm for solution of two-parameter self-adjoint boundary value problems, Zh. Vychisl. Mat. i Mat. Fiz. 31 (5) (1991), 689-697 (in Russian); English transl.: USSR Comput. Math. and Math. Phys. 31 (1991).

[11] T. V. Pak, A study of some singular problems with parameters for systems of ordinary differential equations and computation of spheroidal wave functions, thesis, Vychisl. Tsentr Akad. Nauk SSSR, 1986 (in Russian).

[12] R. G. D. Richardson, Theorems of oscillation for two linear differential equations of the second order with two parameters, Trans. Amer. Math. Soc. 13 (1912), 22-34.

[13] B. D. Sleeman, Singular linear differential operators with many parameters, Proc. Roy. Soc. Edinburgh. Sect. A 71 (1973), 199-232.

[14] L. Turyn, Sturm-Liouville problems with several parameters, J. Differential Equations 38 (3) (1980), 239-259. 